\title{
An Online Collaborative Discussion Platform for Bridging a Technological Reliance Gap in Higher Learning Institutions in Tanzania
}

\author{
Linus John \\ Nelson Mandela - African Institution of Science and Technology, P. O. Box 447, Arusha, Tanzania. \\ Dar es Salaam University College of Education, P. O. Box 2329, Dar es Salaam, Tanzania \\ E-mail: johnl@nm-aist.ac.tz or linusbazz@gmail.com \\ Anael E. Sam \\ Nelson Mandela - African Institution of Science and Technology, P. O. Box 447, Arusha, Tanzania. \\ Email: Anael.Sam@nm-aist.ac.tz or anaelsam@gmail.com
}

Received: 03 September 2018; Accepted: 07 October 2018; Published: 08 November 2018

\begin{abstract}
Currently, the online discussion forums have become the focal point for e-learning in many Higher Learning Institutions (HLIs); this is due to the ubiquitous of Information and Communication Technology (ICT) tools, significant rate and fast growing technology adoption and use in many fields including education. However, developing countries, such as Tanzania, are experiencing technical adoption difficulties, such as limited access to computers, problems with Internet connections as well as the technological reliance gap between tutors and learners; these affect the use of technology in Teaching and Learning (T/L). This study aims to use an Online Discussion Platform (onlineDP) to bridge the technological reliance gap between the tutors and learners in HLIs in Tanzania. In this study, the literature review and qualitative research methods were conducted to develop the prototype of the platform. The UMBC semantic similarity service was used to develop the contents filter used to reduce the number of duplicate discussion questions. The application was mainly developed using Laravel Pre-processor (PHP) framework and My Structured Query Language (MySQL) database. The result is the web-based application prototype that enhances the collaborative learning environment in HLIs in Tanzania. The technologies to be used for $\mathrm{T} / \mathrm{L}$, should consider both sides of tutors and learners as well as the theoretical framework for their implementations.
\end{abstract}

Index Terms-Online Discussion Platform, online Asynchronous Discussion Forum, Online Discussion Group, Web-Based Application, E-Learning.

\section{INTRODUCTION}

It's significantly understood that, in the collaborative learning situation, discussion is an excludable entity that has been increased in the adoption and use of the ICT in the $\mathrm{T} / \mathrm{L}$ processes. This has consequently led to the fast development and use of online platforms in offering an elearning. In the recent past, education system delivery in Tanzania has received adequate changes through the use of learning management systems (LMS) such as Moodle platform [1]. Additionally, the asynchronous discussion forums such as Stackoverflow, Quora, Edmodo, to mention a few and those found into the LMS are used to offer online learning as well as to supplement the classroom T/L. The asynchronous discussion forums provide a virtual collaborative space for collaborating entities and facilitate a free learning environment without limitations of physical availability, geographical settings and time [2]. In addition, asynchronous groups found in social networks (SNs) such as the Facebook, WhatsApp, Twitter, among others, help to facilitate social collaboration, sharing knowledge, strengthening the learning and decision making.

Although ICT is being adopted in T/L environment, there are still many challenges faced globally, for instance, the technical problems such as Internet connection problems, limited computer access and the technology used by the young generation vis-a-vis the old generation which has consequently left a huge gap due to the fact that, an era in which a technology is being invented makes it a native for people born in that time; meanwhile, leaving the other generations as immigrants [3]. The reliance of the technology between the tutors and learners differs in a way that learners engage more in the use of technology that tailors around their area of experience and it differs from the technology used by tutors in the context of e-learning. For example, many tutors in HLIs in Tanzania are trained to use the traditional LMS, whereas, their learners prefer to use new trending technologies such as social networks (SNs) platforms; this makes them active and innovative in learning [1, 4].

Recently, researchers are exploring the technology reliance gap that exists between tutors and learners. Some researchers, propose to have an LMS integrated with the 
SN platform, to enhance efficiency and frequent interaction such as the exchange of informational contents, communication skills building, and cooperation during and after classrooms [4]. Additionally, some scholars suggest the use of LMS integrated with discussion forums in $\mathrm{T} / \mathrm{L}$ as well as $\mathrm{SN}$ groups [5]. However, Tess in [6] argued that, although SN and other educational technologies can support T/L activities, but their uses in educational settings shouldn't solely be accounted for their practical integration, or the extent to which they support attainment of the course goals, but also the focus has to be on the theoretical context towards their implementation as the learning resources.

\section{A. HLIs and ICT adoption and Integration in $T / L$}

The world's education system has been revolutionized by the adoption and integration of ICT tools in T/L; which has improved the cost-effectiveness of higher learning education, meanwhile developing the $21^{\text {st }}$ Century workforce competences and skills of learners for current and future workplace requirements [7,8]. Likewise, if ICT is properly integrated in education, it widens the range of access to education and allows for successful accomplishment T/L objectives as compared to the solely traditional approach [1].

Additionally, Professor Bates, the expert in the field of distance learning and who had worked in the area of online learning [9] pointed out that, online learning has become so popular due to: growing access to, experience plus comfort learners have with technology, the essentiality of flexible programs delivery to learners who are working and learning, in the active learning and collaborative learning perspective, and excessive conventional classrooms.

In [9], it's pointed out, the higher rates of the development, implementation and integration of elearning in the education system in Canada and United States of America (USA); where in USA more than $90 \%$ of its HLIs use LMS with increased online class enrolment and around 11 million of learners are taking some of their classes online. The higher rates are as well reported in United Kingdom (UK), New Zealand, Australia, and Northern Europe, moreover, there has been a very rapid growth of adoption and use in the India and China.

Coming to Africa, Sub-Saharan African countries, although the number of e-learning technology adoption and use is low, but several HLIs in different countries are continuing to adopt and use some LMS [1,9]. For instance, at University of South Africa (UNISA), myUnisa web application is used for collaboration and interaction and the university tutors use Sakai platform for dissemination materials and facilitating the interaction with their learners, while the mobile technology is kept for learners' communications [1,10]. Additionally, other HLIs use the LMS for complimenting the face-to-face $\mathrm{T} / \mathrm{L}$ approaches, for instance, "The University of Johannesburg (UJ) which has over 45,000 full-time, parttime, face-to-face learners spread across seven campuses. It uses LMS to supplement course delivery" [11].

\section{B. ICT adoption and Integration in $T / L$ in Tanzania}

In Tanzania, to obtain higher education is the dream and line of life for many children [1] and their supporters. So far the government through Higher Education Students' Loan Board (HESLB) does it best to offer loans to support learners to pursue their higher education; this has consequently led to an increase in number of intakes in HLIs [12]. But still, HLIs in Tanzania depend much on the conventional setting of the lecture rooms [1], nonetheless, they are challenged by the insufficient number of human capital and physical facilities such as lecture rooms, public places for discussion and library materials [13].

This has in fact, influenced Tanzania to adopt and use different technologies as an effort to expand access as well as to improve the quality of her education. However, the technologies adopted and used in institutions are subjected to high license fees, for instance, institution that uses Blackboard system may be needed to pay a yearly license which is not less that USD $\$ 18,000$, incompatibility with mobile computing devices such as mobile phones for example HLIs that use Moodle need to do an extra customization for it to feet with mobile phones [1].

\section{An Overview of Asynchronous Discussion Group}

An asynchronous discussion group is an aggregate of people who use the asynchronous discussion platform to create a virtual discussion space, to share knowledge, express opinion, ask and/or reply on questions from each other, and to build their capacity. In order to execute the discussion virtually, there must exist a very successful discussion forum. It is well articulated in [2], that, it's neither the abundance of the asynchronous forums for discussion nor the comfortability the tutors and learners make the online collaboration successful, but its successful creation. Thus before embarking on developing or creating one, it's important to study and critically analyze the types of asynchronous forums.

Online discussion forums are principally classified into three classes: The Auxiliary Discussion Forums, The Hybrid Discussion Forums, and The Embedded Discussion Forums, they are respectively given as a supplement to a face-to-face classroom learning model, major component in the face to face model or the sole component of the classroom learning - the course has to be whole online. With respect to the interaction and participation of tutors and learners and activities in the discussion, the three classes were compared and contrasted based on the inclusion of the following points [2]:

- Participation in the discussion

- Instructor's presence

- Major Interaction

- Participation Assessment

- Lecturers Engagement

- Massage Quantity

- Topic Time-To-Live 
- $\quad$ Receiving Feedback

- Learning and

- Activity

These points are distributed among the classes as illustrated using the Venn diagram in Fig. 1. It shows that a hybrid asynchronous discussion forum is allencompassing the others, and it captures all the points; thus it leverages the rest.

Basing on the findings from these researches: the exploration of the technological gap between tutors and learners, the importance of asynchronous group discussion and the asynchronous discussion forums points as in Fig. 1. and findings from the Tanzanian elearning context, an onlineDP was designed and developed, it's a web-based discussion group application which ensures the hybrid asynchronous discussion nature; this allows both tutors and learners to meet virtually in a monitored and controlled learning environment for doing discussion asynchronously as a supplement to the normal classroom $\mathrm{T} / \mathrm{L}$ delivery model. Using onlineDP tutors should be able to create and interact with the virtual groups of learners and assign tasks to discuss, they should as well be capable of tracking the participation of each learner, individually or group wise. The contents in the application has to be moderated by the moderators; moreover, the application uses the semantic similarity search algorithm to limit the number of contents (message quantity), questions or post by reducing the number of duplicates.

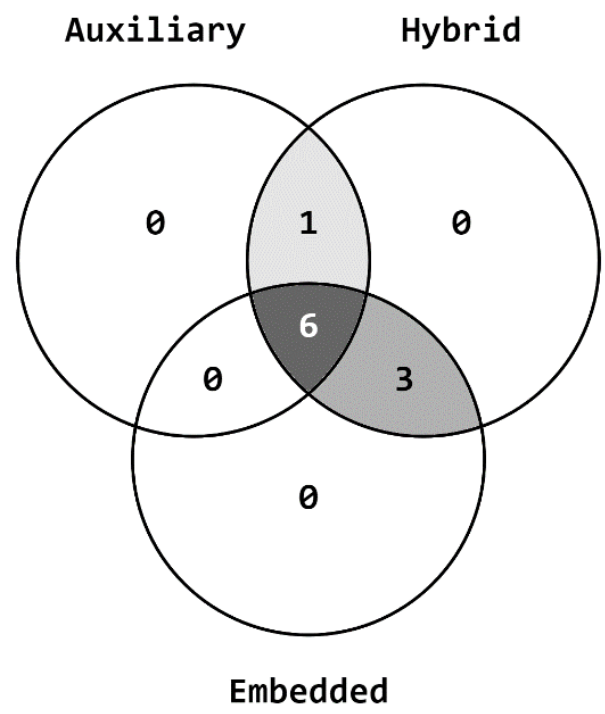

Fig.1. Classification of Asynchronous Discussion Groups

\section{METHODOLOGY}

This study applied documentary literature review and qualitative methods for requirement gathering from different documents, educational stakeholders and Information Technology (IT) personnel from HLIs in
Arusha, Tanzania and then the requirements for the application were specified.

The onlineDP prototype to be developed will use PHP Framework with Bootstrap and MySQL database to make it fast, fluid, easy to use, and a responsive web-based application. It will be accessed via web browsers such as Google Chrome, Firefox, Microsoft Edge.

The duplicate control part uses the algorithm which developed using the UMBC semantic similarity service API [14] to calculate the relevance of the contents to be stored.

\section{REQUIREMENTS SPECIFICATION}

Towards the development of onlineDP, a web application proposed in this study, both non-functional and functional requirements taken into account. Whereas, the functional requirements cover (1) the virtual discussion environment enhancement as the creation and management of groups, asking and answering questions, share of materials and tasks as well as reacting over the contents by means of votes and comments. Tutors and learners, or learners themselves in groups, should be able to carry out their activities asynchronously. (2) Redundancy control, to make sure that information (question) does not exist in multiples, the application should perform a semantic similarity search to the database of questions; whenever equivalent contents are found, it has to collect the relating records and redirect the user to the relevant contents. (3) Content moderation, the moderation has to carried out by moderators to make sure that the information shared or accessed do not violet the Tanzania legal information access and use requirements as defined in section 16 of Cybercrime Act 2015 [15]. The application should allow the user with moderation privilege to traverse the contents.

The non-functional requirements cover issues of system's operability, maintainability, security, and performance

\section{Designing THE PROPOSED APPLICATION}

This section includes the data flow diagram that indicates how the requirement have been modeled, the complete system design, the system's main operations and it explains different tools that used to develop the system and the reason for their selection over others, and some key application's intefaces.

\section{A. Data-flow diagrams (DFDs)}

These are system models which show a system's functional perspective; the transformations represent processes or functions. They are used to show a sequence through which data has to pass in processing steps. For example, a processing step could be the filtering of duplicate records in a customer database. The DFD in Fig. 2. Shows different external entities represented by the rectangles, these interact with the onlineDP application. The processes and transformations show data flows and 
processes that may be taken on them. Moreover, it shows the scope of the application $[8,17]$.

\section{B. Web-based application}

Web-based applications also known as web apps include all programs which run inside web browsers, are accessible via hypertext-transfer-protocol (HTTP) network connections [18]. This study applies Laravel PHP framework with Bootstrap for frontend and the MySQL database for backend to allow the onlineDP to be a fast, secure and responsive web app, an application that may respond differently when accessed by devices with different viewports. Thus sufficing the needs of users with different computing devices.

\section{1) Bootstrap}

Bootstrap was developed by Jacob Thornton and Mark Otto; it was initially released when they were both employees of Twitter. It is an open source component for building software with Hyper Text Markup Language (HTML), JavaScript (JS), and Cascading Style Sheet (CSS), Bootstrap enables for responsive web design $[19,20]$
Why use Bootstrap: Laravel is by default released with the Bootstrap, enables to take the advantage of Bootstrap features, and thus quickens the process of building the application prototype by using its mixins and Sass variables, responsive-grid system, extensive prebuilt gears, and powerful JQuery plugins [19]. In addition, Bootstrap ensures that a web application is responsive to different viewports. This makes onlineDP accessible by a large range of computing devices.

\section{2) Laravel PHP Framework}

Laravel PHP framework is an MVC (Model-ViewController) framework for web-development written in PHP scripting language released under MIT license, it has its source code on GitHub [21]. The model section has been used for managing every task related to data, the model basically deals with data access abstraction handling and validation. The View was used as the user interface; it's mainly built by an HTML, CSS and JS template so that it enables the users of the application to interact with it. Moreover, Controllers used to handle the user requests to the application and processes the responses in return, it's built of functions which act as triggers of events or event handlers [21-23].

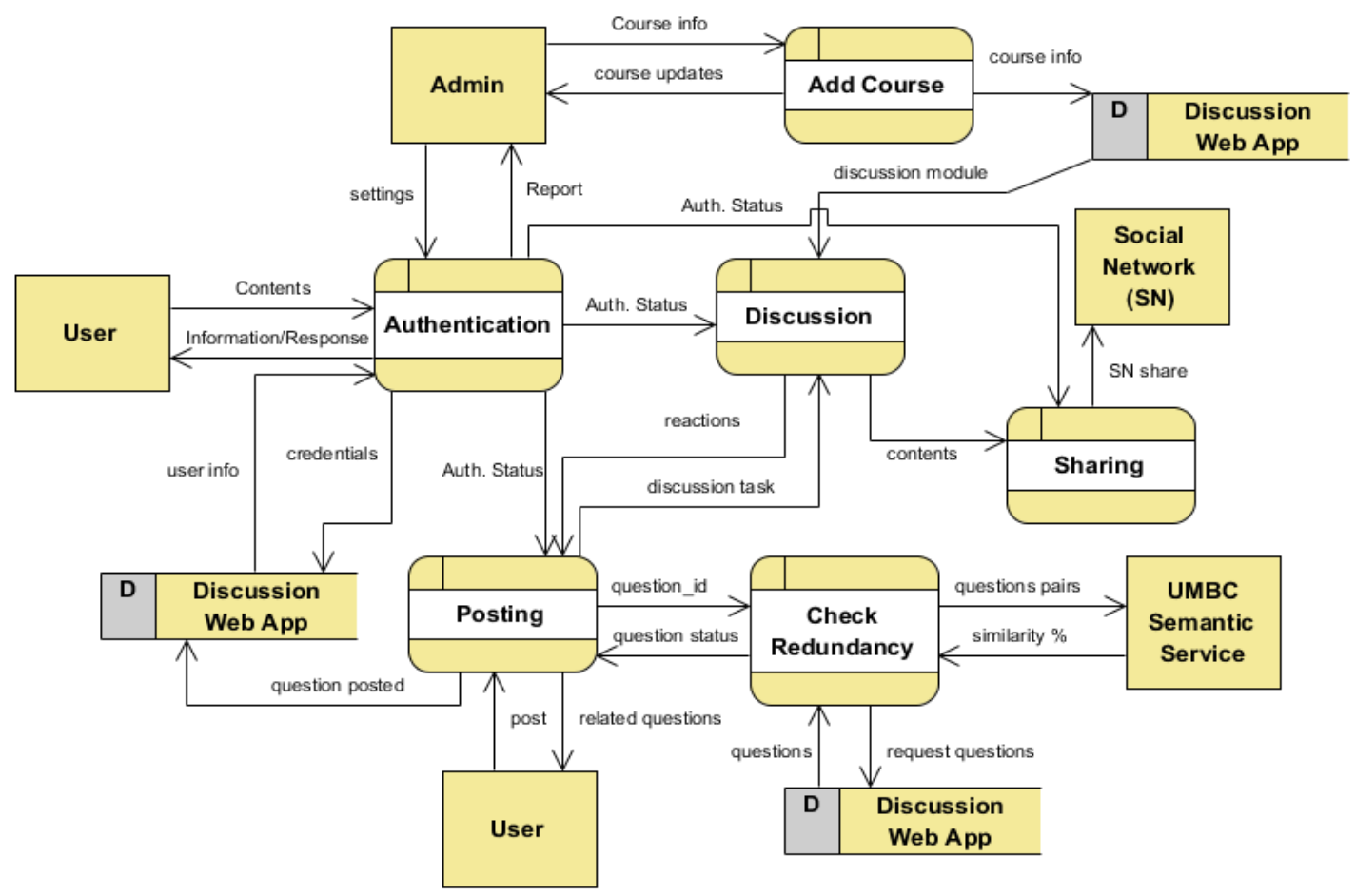

Fig.2.Data-Flow Diagram for The Proposed Web-Application

Laravel makes smart predictions of what a coder is aiming to accomplish, thus assisting write fewer codes, meanwhile achieving the complete goal. Laravel 5.6 which is the current version was used to develop this application.

Why choose Laravel: It's chosen because it supports MySQL database. Contemporarily, Laravel supports these databases: MySQL, MSSQL, PostgreSQL, SQLite and SQL server [24,25] with MySQL as default database. Additionally, as an MVC framework, Laravel is convenient for web applications which are getting accessed and used as the result of HTTP requests and responses [23].

Through the MVC nature of Laravel, it ensures the non-functional requirements of an application are achieved $[21,22,26]$ :

Improves the code maintenance with reduced complexity, in Laravel, different views can be attached to a single data without affecting the activeness of the codes and allows independence of data presentations. 
Increases reusability, from the driver-bundle system combination, it offers a real code modularity. By utilizing bundle, it is possible to package codes for reuse.

Scalability. Laravel is transparent in its data and view presentation, thus it's very easy to add or remove components from the application.

Security, Laravel always applies the highest and most advanced PHP technologies. It uses its Hash class to give a secure Bcrypt for securing passwords.

$$
\mathbb{Z} \$ \text { password }=\text { Has }: \text { :make('yourpassword' ) }
$$

Moreover, Laravel 5 comes with HTTP middleware which increases security by offering a freedom to create customized security mechanisms besides the Laravel's default authentication mechanism [25].

Why prefer a PHP framework: because PHP is the most used server-side scripting language for web application development and is an open source. Additionally, PHP runs in its own memory space, thus having quicker load time; this makes PHP codes to run fast with increased application's efficiency [27].

\section{3) $M y S Q L$}

MySQL differs from other database servers and has the architectural features that make it somewhat general purpose. It's very flexible and works well in highly demanding environments like in web application [28]. MySQL is the application that runs on a server, and it uses standardized Structured Query Language. Additionally, it has varieties of storage engines which support different application demands.
Why MySQL: It is supported by the development framework used in this study, and it's the default database configuration that Laravel ships with. When opening up the database.php file located at ...//project_folder//config, there is the following line; 'default' => env('DB_CONNECTION', 'mysql'), hence using MySQL costs nothing in database configurations.

With MySQL it's possible to protect data via its unique security features, it's with very strong mechanisms, which ensure that database server access is not only possible but also be for the authorized users. It comes with granular object privilege framework; this ensures that a user only gets and sees all that s/he is supposed to get. Importantly, MySQL has powerful features for data encryption \& decryption functions, which protect unauthorized user access to sensitive data; it uses its Secure Sockets Layer and Secure Shell to offer very secure and safe connections [27]. Moreover, MySQL database has the utilities of automatic recovery and online backup, hot backup [28].

\section{B. Proposed system Design}

\section{1) System Operations}

The onlineDP application (Fig. 3.) operates as follows:

(1 a) User uploads course notes or task.

(1 b) User asks a question.

(2 a) \& (2 b) The 'query processing' combines the question and some records from the database, for each pair, is sent to UMBC semantic similarity services [14] for semantic comparison and calculation.

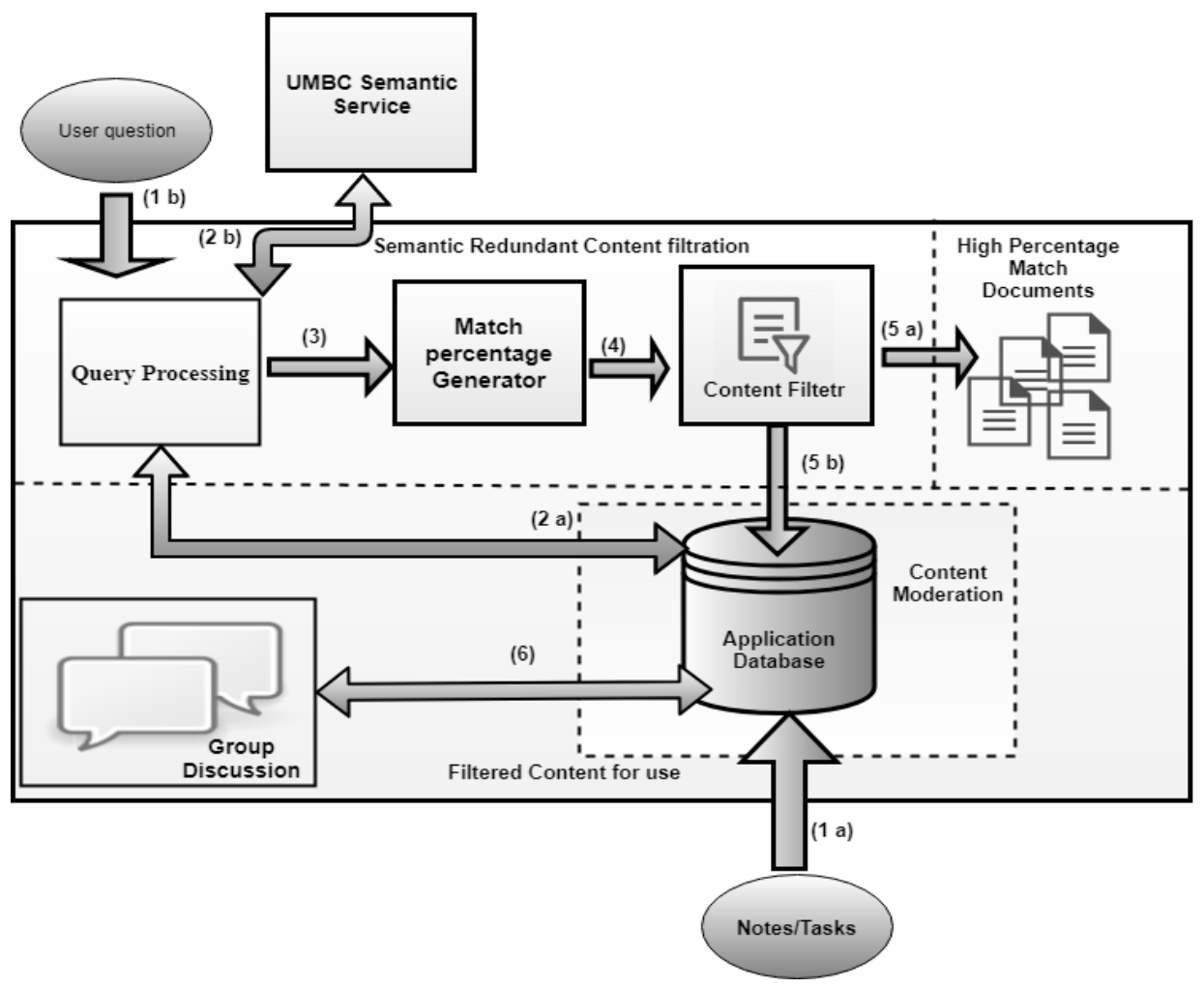

Fig.3. Proposed Online Discussion Platform 
(3) The percentage match is generated.

(4) The system filters each pair of a question and the content, then decides on whether to allow the post to go into the database or to discard and direct the user to the suggested similar documents.

( 5 a) Documents similar to a question 'high percentage matches' is displayed to the user asked the question.

(5 b) If a question appears to have no match or has low percentage match it is then moved to the database for moderation.

(6). The discussion is conducted in the moderated way.

\section{Application Frontend}

The onlineDP is a responsive discussion group web app that works in all modern browsers, in both computers and mobile. The frontend of the application will have several interfaces such as Student's dashboard, Tutor's dashboard and Moderator's dashboard interfaces; these will allow the application's stakeholders, learners, tutors, and moderators to interact on a single shared repository of information.

All onlineDP's interfaces will be developed using Blade, a very powerful Laravel's templating enigine [29], which will be integrated with the bootstrap template to generate the responsive layouts of the application. The icons will be used from the Font Awesome, the iconic and css toolkit with customizable and scallable vector icons [30]. Each user will be able to see menus allowed to by the application's administrator, the menu which will represent a link to an interface which can allow a particular user to perform or get a service from the application.

\section{CONCLUSION}

This study through literature review, it was observed that, there is an increased adoption and use of different technologies in T/L, and the online discussions are the focal points of an e-learning; these have made many HLIs globally to opt for these technologies. Despite the roles played by technology in attaining course goals or facilitating the $\mathrm{T} / \mathrm{L}$ as well as increasing access to education, it has left a very huge gap between tutors and learners, and some of the technologies suggested such as SNs were not primarily developed as the T/L tools.

Following the contextualization of the hybrid asynchronous features to the HLIs in Tanzania on the basis of legal and HLIs ICT level of adoption, this study has therefore, used Laravel PHP framework, Bootstrap and MySQL to developed the hybrid asynchronous discussion platform, the responsive web-based app that will allow the virtual collaboration between the tutors and learners of all technological epochs. It uses the semantic similarity algorithm to limit the contents duplicates, and it allows the content moderators to traverse thorough and check the compliance of the information to the cyberspace legal requirements.

The use of the technologies which overgenerous base on one side of either tutors or learners or those which were not primarily built for T/L has to be replaced by the technologies which bridge the overgenerous nature of technologies and consider the theoretical frameworks in the fields where they are going to implemented

\section{REFERENCES}

[1] J. S. Mtebe and C. Raphael, "A decade of technology enhanced learning at the University of Dar es Salaam, Tanzania: Challenges, achievements, and opportunities," Int. J. Educ. Dev. using Inf. Commun. Technol. (IJEDICT), 2017, Vol. 13, Issue 2, pp. 103-115 A, vol. 13, no. 2, pp. 103-115, 2017.

[2] T. K. H. Adeli and H. K. H. Kang, Software Engineering, Business Continuity, and Education, vol. 257. 2011.

[3] E. N. O'Reilly, "Developing technology needs assessments for educational programs: An analysis of eight key indicators," Int. J. Educ. Dev. using Inf. Commun. Technol., vol. 12, no. 1, pp. 129-143, 2016.

[4] G. Mozhaeva, A. Feshchenko, and I. Kulikov, "E-learning in the Evaluation of Students and Teachers: LMS or Social Networks?" Procedia - Soc. Behav. Sci., vol. 152, pp. 127-130, Oct. 2014.

[5] H. Meishar-Tal, G. Kurtz, and E. Pieterse, "Facebook groups as LMS: A case study," Int. Rev. Res. Open Distance Learn., vol. 13, no. 4, pp. 33-48, 2012.

[6] P. A. Tess, "The role of social media in higher education classes (real and virtual)-A literature review," Comput. Human Behav., vol. 29, no. 5, pp. A60-A68, 2013.

[7] T. Bates, "Expectations and goals for e-learning," 2009. [Online].

Available: https://www.tonybates.ca/2009/06/18/expectations-andgoals-for-e-learning/. [Accessed: 10-Oct-2018].

[8] E. Dobozy and P. Reynolds, "From LMS to VLE or from supermarkets to airports: Classifying elearning platforms using metaphors," Proc. 5th Int. LAMS Conf. 2010, pp. 92-103, 2010.

[9] A. Simsek, "Interview with Tony Bates on the Aspects and Prospects of Online Learning," Contemp. Educ. Technol., vol. 2, no. 1, pp. 88-94, 2011.

[10] P. Venter, M. J. V. Rensburg, and A. Davis, "Drivers of Learning Management System Use in a South African Open and Distance Learning Institution," Australas. J. Educ. Technol., vol. 28, no. 2, pp. 183-198, 2012.

[11] E. Bagarukayo and K. Billy, "Evaluation of elearning usage in South African universities: A critical review Billy Kalema," Int. J. Educ. Dev. using Inf. Commun. Technol. (IJEDICT), 2015, Vol. 11, Issue 2, pp. 168-183, vol. 11, no. 2, pp. 168-183, 2015.

[12] URT, "National Basic Education Statistics in Tanzania (BEST)," 2016.

[13] S. S. Yeni Murdani Istoroyekti and M. Hum, "ISSUES CHALLENGING UNIVERSITIES: A CASE OF," Ahmad Dahlan J. English Stud., vol. 3, no. 1, pp. 51-62, 2016.

[14] L. Han, A. Kashyap, T. Finin, J. Mayfield, and J. Weese, "UMBC_EBIQUITY-CORE: Semantic Textual Similarity Systems," Proc. 2nd Jt. Conf. Lex. Comput. Semant., vol. 1, pp. 44-52, 2013.

[15] URT, Tanzania Cybercrimes Act, 2015, vol. 96, no. 14. 2015, pp. 25-26.

[16] K. E. Kendall and J. E. Kendall, Systems Analysis and Design, Ninth Edit. New York: Pearson Education, Inc., 2011.

[17] I. Sommerville, Software Engineering. 2010.

[18] "Web-Based Application." [Online]. Available: https://www.techopedia.com/definition/26002/web-basedapplication. [Accessed: 28-Jul-2018]. 
[19] "Bootstrap." [Online]. Available: https://getbootstrap.com/. [Accessed: 27-Jul-2017].

[20] J. Spurlock, Bootstrap: Responsive Web Development, First Edit. New York: O’Reilly Media, Inc., 2013.

[21] R. F. Olanrewaju, T. Islam, and N. Ali, "Advanced Computer and Communication Engineering Technology," vol. 315, 2015, pp. 399-410.

[22] M. Kalelkar, P. Churi, and D. Kalelkar, "Implementation of Model-View-Controller Architecture Pattern for Business Intelligence Architecture," Int. J. Comput. Appl., vol. 102, no. 12, pp. 975-8887, 2014.

[23] D. P. Pop and A. Altar, "Designing an MVC model for rapid web application development," Procedia Eng., vol. 69, pp. 1172-1179, 2014.

[24] "Laravel." [Online]. Available: https://laravel.com/docs/5.6/database. [Accessed: 27-Jul2018].

[25] S. McCool, Laravel Starter - The definitive introduction to the Laravel PHP web development framework, no. Book, Whole. 2012.

[26] M. R. J. Qureshi and F. Sabir, "a Comparison of Model View Controller and Model View Presenter.," Sci. Int., vol. 25, no. 1, pp. 7-9, 2013.

[27] N. Mduma, "An Integrated Mobile Application for Enhancing Management of Nutrition Information in Arusha," Int. J. Comput. Sci. Inf. Secur., vol. 13, no. 7, pp. 34-38, 2015.

[28] B. Schwartz, P. Zaitsev, and V. Tkachenk, High Performance MySQL, Third Edit. Gravenstein Highway North, Sebastopol: O’Reilly Media, Inc., 2012.

[29] "Blade Templates." [Online]. Available: https://laravel.com/docs/5.6/blade\#introduction.

[Accessed: 17-Oct-2018].
[30] "Font Awesome." [Online]. Available: https://fontawesome.com/v4.7.0/. [Accessed: 17-Oct2018].

\section{Authors' Profiles}

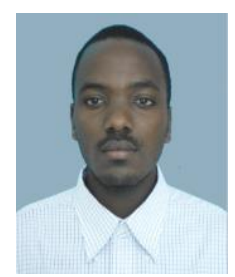

Linus John obtained his Bachelor's Degree of Science (Mathematics and Informatics) with Education in 2015 from UDSM at Dar es Salaam University College of Education (DUCE), a constituent college of UDSM.

He joined DUCE as Informatics academic staff early in 2016. He has passionate in ICT related researches especially on Integration of ICT in Education. Currently, he is pursuing Master's Degree in Information and Communications Science and Engineering (ICSE) at the Nelson Mandela - African Institution of Science and Technology (NM-AIST).

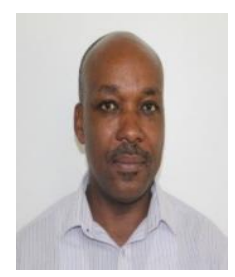

Anael E. Sam is a senior lecturer at the Nelson Mandela African Institution of Science and Technology (NM-AIST, Arusha, Tanzania. He is a holder of Bachelor, Master's and $\mathrm{PhD}$ in Electronics from the Slovak University of Technology in Bratislava, Slovak Republic. His interests are cyber security, mobile communication systems and ICT for development.

How to cite this paper: Linus John, Anael E. Sam, " An Online Collaborative Discussion Platform for Bridging a Technological Reliance Gap in Higher Learning Institutions in Tanzania", International Journal of Modern Education and Computer Science(IJMECS), Vol.10, No.11, pp. 29-35, 2018.DOI: 10.5815/ijmecs.2018.11.04 